\title{
ON THE SCHRÖDINGER EQUATION WITH EXPONENTIAL NONLINEARITY *
}

\author{
J.P. SHEERIN \\ Department of Physics, University of Michigan, Ann Arbor, Michigan 48109, USA \\ and \\ R.S.B. ONG \\ Department of Aerospace Engineering, University of Michigan, Ann Arbor, Michigan 48109, USA
}

Received 20 June 1977

\begin{abstract}
The Schrödinger equation with exponential nonlinearity is solved in the time-independent case. The solutions are compared with those obtained from the case with cubic nonlinearity.
\end{abstract}

Consider a large amplitude electromagnetic wave of frequency $\omega_{0}$ incident on the critical surface (where $\omega_{0}=\omega_{\mathrm{p}}$, the electron plasma frequency) of a dense plasma. The ponderomotive force generated (in this region) acts to expel matter from the region of high field intensity forming a density depression. The depression serves to trap the field, enhancing the ponderomotive force which in turn deepens the cavity. The ponderomotive force and consequent density cavity allow the field to penet rate the critical surface where $\omega_{0}<\omega_{\mathrm{p}}$, and the dielectric permittivity

$\epsilon=1-\left(\omega_{\mathrm{p}}^{2} / \omega_{0}^{2}\right)$,

is negative.

The field in the density cavity is described by the Maxwell's equations coupled with the equations of continuity and motion for the particles. A straightforward calculation leads to a wave equation with a density dependent term [1]. In one dimension this may be written as

$\frac{\partial^{2} E}{\partial t^{2}}-c^{2} \frac{\partial^{2} E}{\partial x^{2}}=-\omega_{\mathrm{p}}^{2}\left(n / n_{0}\right) E$,

where $\boldsymbol{E}$ is the transverse electric field and $n_{0}$ is the ambient plasma density.

The density dependent coefficient is, in turn, dependent on the field. The time-averaged force acting on an electron in a rapidly oscillating electromagnetic field is

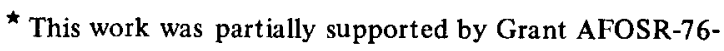
2904.
$\boldsymbol{F}=-\nabla\left(e^{2} / 4 \pi m \omega_{0}^{2}\right)|E|^{2}=e \phi$,

where $\phi$ is the ponderomotive potential. Assuming thermal equilibrium, the electron and ion densities are

$n_{\mathrm{e}}=n_{0} \exp \left[e\left(\phi+\phi_{\mathrm{s}}\right) / T_{\mathrm{e}}\right], \quad n_{\mathrm{i}}=n_{0} \exp \left[-e \phi_{\mathrm{s}} / T_{\mathrm{i}}\right]$,

where $\phi_{\mathrm{s}}$ is the electromagnetic potential due to charge separation. If the scale length $L \equiv\left[\nabla \ln \left(|E|^{2}\right)\right]^{-1}$ $\gg \lambda_{\mathrm{D}}$, the electron Debye length, we may set $n_{\mathrm{e}}=n_{\mathrm{i}}$, and obtain

$n_{\mathrm{e}}=n_{\mathrm{i}}=n_{0} \exp \left[-e^{2}|E|^{2} / 4 \pi m \omega_{0}^{2}\left(T_{\mathrm{e}}+T_{\mathrm{i}}\right)\right]$,

which is exponentially dependent on the square of the amplitude of the field.

In many treatments of the problem the exponential term is usually expanded and higher order terms in the series expansion are then neglected. The consequences of such a truncation are, in part: the density

$n / n_{0} \simeq 1-\left[e^{2}|E|^{2} / 4 \pi m \omega_{0}\left(T_{\mathrm{e}}+T_{\mathrm{i}}\right)\right]$,

becomes unphysical as $|E|$ grows, in contrast to expression (5) which decreases exponentially as the field increases; and the dielectric permittivity in the expanded version increases without bounds with increasing amplitude of the electric field. On the other hand the inclusion of the exponential forces it to saturate at large values in agreement with observed phenomena in experiments in nonlinear optics. Hence we are motivated to retain the full exponential nonlinearity when considering fields of significant amplitude.

We separate the field into a slowly-varying envelope modulated by a high frequency oscillation 
$E(x, t)=E_{0}(x, t) \exp \left(-\mathrm{i} \omega_{0} t\right)$.

Substituting this into eq. (2) and neglecting the higher order time derivative we obtain the nonlinear Schrödinger equation for the envelope

$2 \mathrm{i} \frac{\partial E_{0}}{\partial t}+c^{2} \frac{\partial^{2} E_{0}}{\partial x^{2}}-(\nu+\sigma) E_{0}=0$

where

$\sigma=\left(\omega_{\mathrm{p}}^{2}-\omega_{0}^{2}\right) / \omega^{2}, \quad \nu=\left(n-n_{0}\right) / n_{0}$,

and $E_{0}$ indicates the amplitude of the slowly varying envelope. We can interpret $\sigma$ as a nonlinear frequency shift.

We now introduce the dimensionless variables:

$$
\begin{gathered}
\tau=1 / 2\left(\omega_{\mathrm{p}}^{2} / \omega_{0}\right) t, \quad \tilde{x}=\left(\omega_{\mathrm{p}} / c\right) x, \\
|\widetilde{E}|^{2}=\left|E_{0}\right|^{2} / 4 \pi n_{0}\left(T_{\mathrm{e}}+T_{\mathrm{i}}\right) .
\end{gathered}
$$

In these dimensionless variables eq. (8) becomes

$\mathrm{i} \frac{\partial \widetilde{E}}{\partial \tau}+\frac{\partial^{2} \widetilde{E}}{\partial \tilde{x}^{2}}-(\nu+\sigma) \tilde{E}=0$.

We now let $\xi \equiv \tilde{x}-M T$, where $M$ is the Mach number with the ion-acoustic speed as reference and separate the envelope into a modulus and phase, both dependent on the moving coordinate $\xi$,i.e.

$\widetilde{E}=u(\xi) \exp [\mathrm{i} \phi(\xi)]$.

Substituting this into eq. (11) and integrating once, we obtain an equation for the modulus $u(\xi)$ which is analogous to an "energy" equation in classical mechanics $\left(u^{\prime}\right)^{2}+K u+\exp \left(-u^{2}\right)=A$, where $K \equiv\left(\omega_{0}^{2} / \omega_{\mathrm{p}}^{2}\right)$ $+1 / 4 M^{2}$, and prime means differentiation with respect to the argument. We note that $V(u) \equiv K u^{2}+$ $\exp \left(-u^{2}\right)$ is the "potential energy" well. The energy level in the well is determined by the constant of integration $A$. For (i) $A>1$, the envelope oscillates about zero, (ii) $A<1$, the envelope oscillates about some value other than zero, and (iii) $A=1$, the envelope is a solitary pulse or "soliton". The frequency shift $\sigma$ is plotted as a function of the normaiized intensity $x=u^{2}$ in fig. 1 .

In the regime of current experimental conditions all three types of solutions may be observed. At large intensities $(x>1)$ anticipated in laser fusion devices the oscillatory envelope of alternating phase would be expected to dominate.

In fig. 2 we show a comparison of the solutions ob-

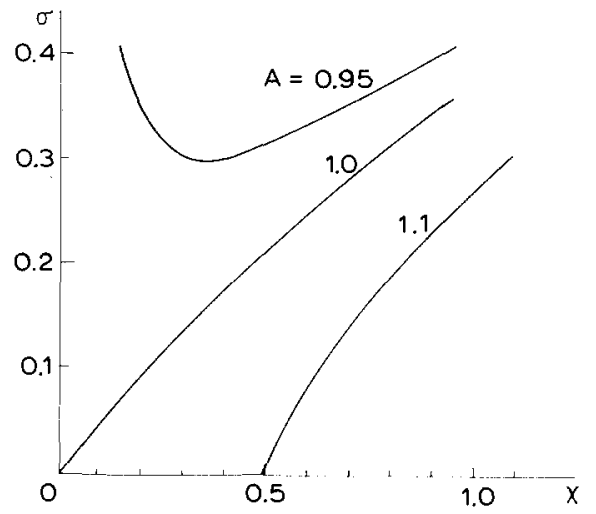

Fig. 1. Frequency shift versus the normalized field intensity.
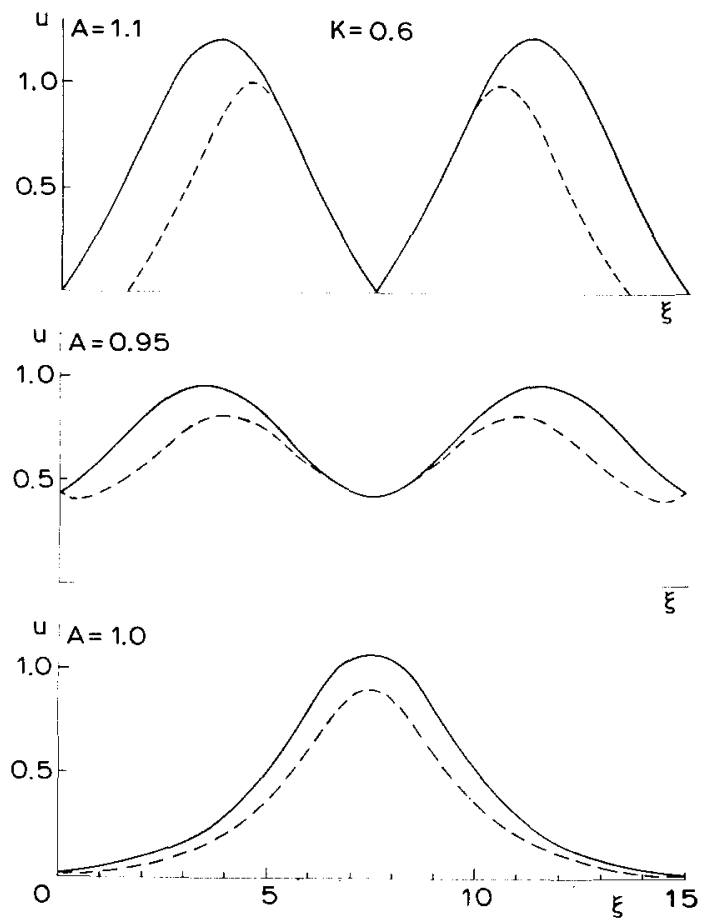

Fig. 2. Comparison of the forms of solutions for the cubic $(--)$ ) and exponential (-) nonlinearities with $K=0.6$.

tained in the expanded, weakly nonlinear case (cubic nonlinearity) and the case with the exponential nonlinearity. The form of the solution in each case is preserved although the exponential solution is larger in both amplitude and width. The value of $K=0.6$ is used in obtaining the figures.

This work has been partially supported by Grant AFOSR-76-2904.

[1] V.E. Zakharov, JETP (USSR) 62 (1972) 1475. 